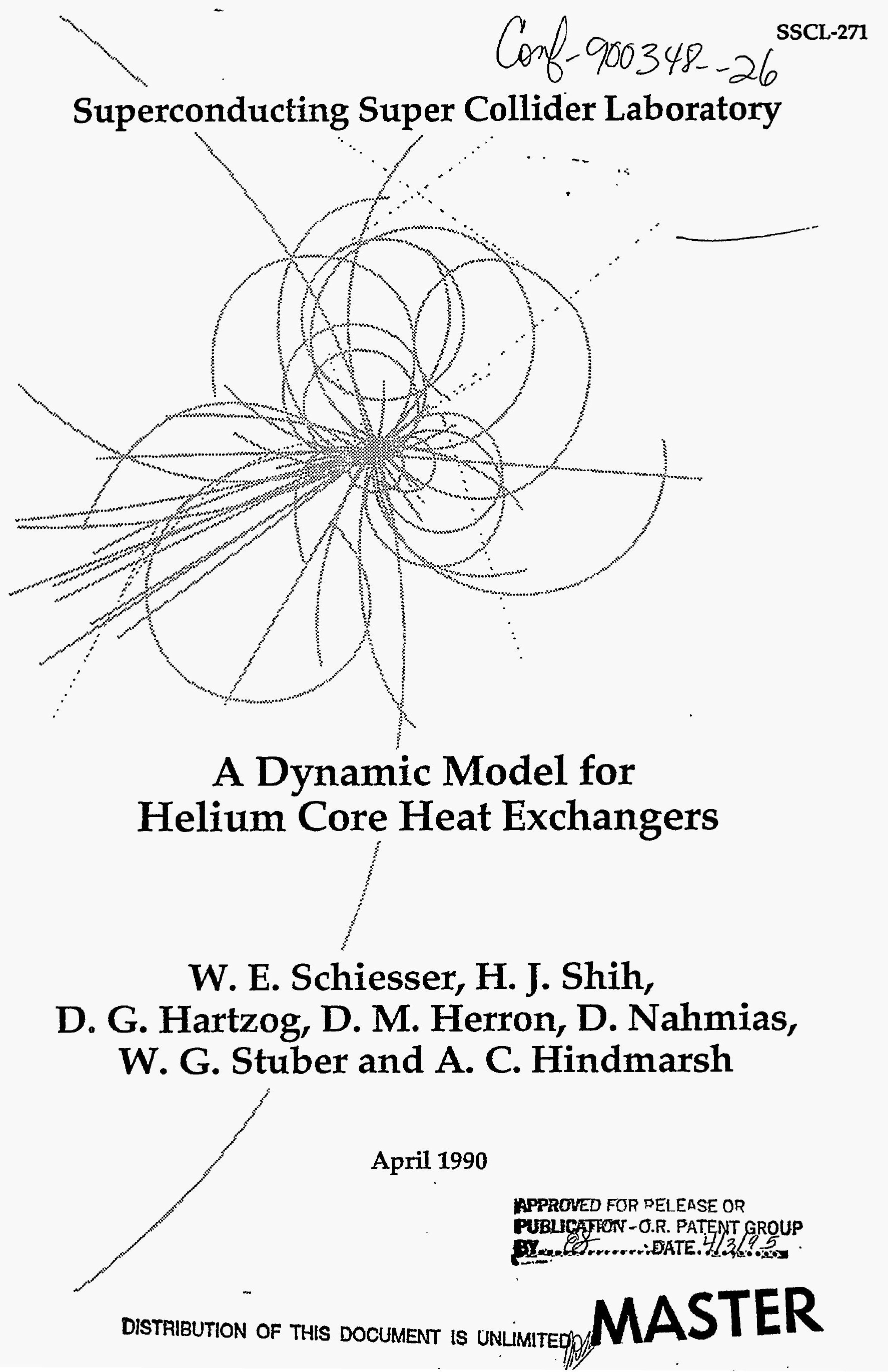




\title{
A DYNAMIC MODEL FOR HELIUM CORE HEAT EXCHANGERS•
}

\author{
W. E. Schiesser \\ Lehigh University \\ Bethlehem, PA 18015 \\ and \\ Superconducting Super Collider Laboratory ${ }^{\dagger}$ \\ Dallas, TX 75237
H. J. Shih
Superconducting Super Collider Laboratory ${ }^{\dagger}$
Dallas, TX 75237

D. G. Hartzog, D. M. Herron, D. Nahmias and W. G. Stuber Air Products and Chemicals, Inc.

Allentown, PA 18195

A. C. Hindmarsh

Lawrence Livermore National Laboratory

Livermore, CA 94550

April 1990

- Presented at the International Industrial Symposium on the Super Collider, Miami Beach, Florida, March 14-16, 1990.

t Operated by the Universities Research Association, Inc., for the U.S. Department of Energy under Contract No. DE-AC02-89ER40486.

\section{DISCLAIMER}

This report was prepared as an account of work sponsored by an agency of the United States Government. Neither the United States Government nor any agency thereof, nor any of their employees, makes any warranty, express or implied, or assumes any legal liability or responsibility for the accuracy, completeness, or usefulness of any information, apparatus, product, or process disclosed, or represents that its use would not infringe privately owned rights. Reference herein to any specific commercial product, process, or service by trade name, trademark, manufacturer, or otherwise does not necessarily constitute or imply its endorsement, recommendation, or favoring by the United States Government or any agency thereof. The views and opinions of authors expressed herein do not necessarily state or reflect those of the United States Government or any agency thereof. 


\section{DISCLAIMER}

Portions of this document may be illegible in electronic image products. Images are produced from the best available original document. 


\title{
A DYNAMIC MODEL FOR HELIUM CORE HEAT EXCHANGERS
}

\author{
W. E. Schiesser \\ Lehigh Üniversity, Bethlehem, PA 18015 \\ and \\ Superconducting Super Collider Laboratory, ${ }^{*}$ Dallas, TX 75237 \\ H. J. Shih \\ Superconducting Super Collider Laboratory," Dallas, TX 75237 \\ D. G. Hartzog, D. M. Herron, D. Nahmias and W. G. Stuber \\ Air Products and Chemicals, Inc., Allentown, PA 18015 \\ A. C. Hindmarsh \\ Lawrence Livermore National Laboratory, Livermore, CA 94550
}

\begin{abstract}
To meet the helium ( $\mathrm{He}$ ) requirements of the superconducting supercollider (SSC), the cryogenic plants must be able to respond to time-varying loads. Thus the design and simulation of the cryogenic plants requires dynamic models of their principal components, and in particular, the core heat exchangers. In this paper, we detail the derivation and computer implementation of a model for core heat exchangers consisting of three partial differential equations (PDEs) for each fluid stream (the continuity, energy and momentum balances for the $\mathrm{He}$ ), and one PDE for each parting sheet (the energy balance for the parting sheet metal); the PDEs have time and axial position along the exchanger as independent variables. The computer code can accommodate any number of fluid streams and parting sheets in an adiabatic group. Features of the code include: rigorous or approximate thermodynamic properties for $\mathrm{He}$, upwind and downwind approximation of the PDE spatial derivatives, and sparse matrix time integration. The outputs from the code include the time-dependent axial profiles of the fluid He mass flux, density, pressure, temperature, internal energy and enthalpy. The code is written in transportable Fortran 77 , and can therefore be executed on essentially any computer.
\end{abstract}

- Operated by the Universities Research Association, Inc., for the U.S. Department of Energy under Contract No. DE-AC02-89ER40486. 


\section{Introduction}

The conceptual design of the SSC calls for ten liquid He refrigeration plants to be located at one-tenth intervals around the 53-mile ring (Ref. 1). A basic feature of the operation of the refrigeration plants is related to the unsteady state or dynamic operation of the SSC; typically it will go through transient periods of operation, such as cool down, operation as a particle accelerator, then limited shutdown for maintenance and preparation for the next experiments. Also, unexpected disturbances will occur, such as magnet quenches, that must be accommodated. Because of the low temperatures required for superconducting magnets, several heat shields will be used which require coolant streams at different temperatures. These streams interact through a series of heat exchangers in the refrigeration plants to achieve desired coolant temperatures. The heat exchangers are therefore a major component of the refrigeration plants, and the design of the heat exchangers is a central consideration in determining the operating performance of the refrigeration plants. The dynamic nature of the operation of the refrigeration plants to accommodate normal and unexpected transient conditions within the SSC therefore requires that the modelling and computer simulation of the heat exchangers involve the solution of unsteady-state (time-dependent) partial differential equations.

Core (or plate-fin) heat exchangers are used for the SSC refrigeration plants, mainly for their high heat transfer efficiency and relatively low cost. The heat transfer section of the prototypical core heat exchangers consists of parallel flat plates of metal (called parting sheets), with corrugated metal connecting the plates (called fins). He flows past the fins parallel to the parting sheets, cocurrently or countercurrently. Figure 1 illustrates the stacking of parting sheets and fins and a parallel flow pattern in a core heat exchanger. The passage arrangement for the prototypical core heat exchangers is periodic, i.e., an adiabatic group consisting of a small number of passages is repeated many times, and the adiabatic group has a mirror symmetry about the middle passage. Figure 2 illustrates the passage arrangement within an adiabatic group consisting of four fluid streams. It also illustrates the indexing convention in our model for streams and parting sheets, i.e., the index increments toward the symmetry passage. Our model for the prototypical core heat exchangers is focused on the dynamic behavior of the fluid streams and parting sheets in an adiabatic group.

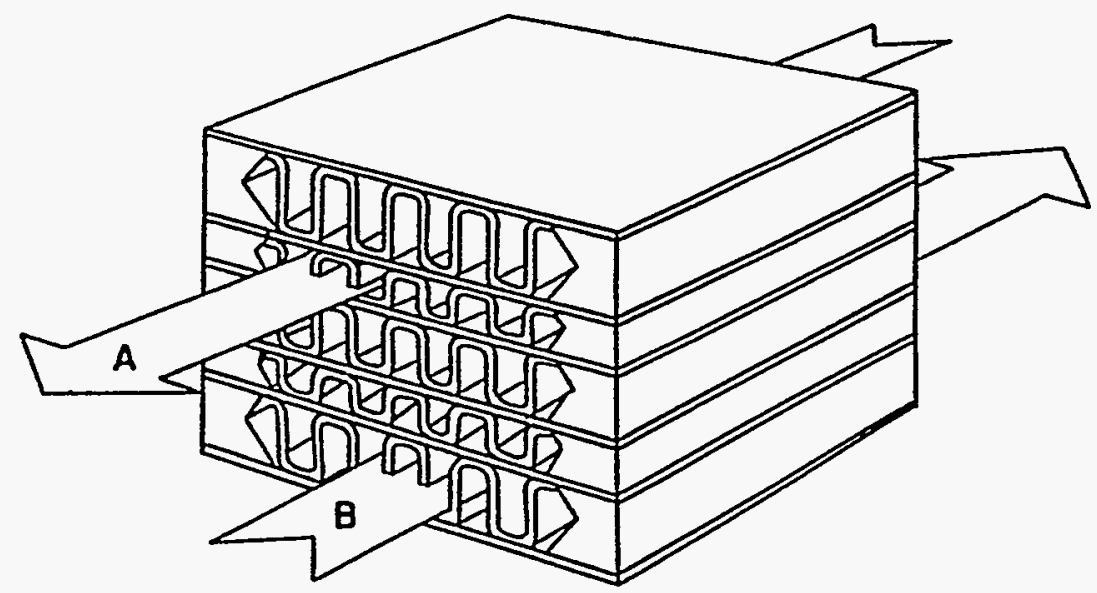

Figure 1: Stacking pattern of parting sheets and fins in a prototypical core heat exchanger. The He streams are flowing parallel to the parting sheets. 
Equation (3) is the momentum balance in Lagrangian form, whereas Eqs. (1) and (4) are in Eulerian form. To have the momentum balance in Eulerian form, we apply the transformation between Lagrangian and Eulerian coordinates

$$
\frac{d v}{d t}=\frac{\partial v}{\partial t}+v \frac{\partial v}{\partial z}
$$

to Eq. (3) and obtain, using the definition $w=\rho v$ and Eq. (1), the time derivative of the He mass flux $w$

$$
\frac{\partial w}{\partial t}=-\frac{\partial P}{\partial z}-\frac{\partial}{\partial z}\left(\frac{w^{2}}{\rho}\right)+F_{e x t}
$$

In our model $F_{\text {ext }}$ includes friction and gravitation, and is thus given by

$$
F_{e x t}=-\frac{f}{2 D} \frac{|w| w}{\rho}-\rho g
$$

where $f$ is the friction factor, $D$ the hydraulic diameter and $g$ the gravitational acceleration. Substituting Eq. (6) for $F_{e x t}$ in Eq. (5) then gives

$$
\frac{\partial w}{\partial t}=-\frac{\partial P}{\partial z}-\frac{\partial}{\partial z}\left(\frac{w^{2}}{\rho}\right)-\frac{f}{2 D} \frac{|w| w}{\rho}-\rho g
$$

The friction factor $f$ is calculated from the Blasius formula for smooth pipes (Ref. 2), i.e.,

$$
f=\frac{0.316}{R_{e}^{0.25}}, \quad R_{e}=\frac{D w}{\mu}
$$

where $\mu$ is the He viscosity. We apply a correction to $f$ to account for the effect of the rough corrugated flow passages.

Applying the energy balance to one parting sheet for longitudinal heat conduction, we obtain the time derivative of the parting sheet temperature $\mathcal{T}_{m}$

$$
\frac{\partial T_{m}}{\partial t}=\frac{1}{\rho_{m} c_{m}}\left\{\frac{\partial}{\partial z}\left(k_{m} \frac{\partial T_{m}}{\partial z}\right)+\frac{Q_{m}}{S_{m}}\right\}
$$

where $\rho_{m}, c_{m}, k_{m}$, and $S_{m}$ are the density, specific heat, thermal conductivity and crosssectional area of the parting sheet, respectively, and $Q_{m}$ is the heat transfer rate per unit length to the parting sheet. The need for determining the temporal dependence of the parting sheet temperature is obvious since streams of different temperatures exchange heat through parting sheets.

Equations (1), (4), (7), and (9) are then the PDEs in our dynamic model for core heat exchangers, and the He density $\rho$, internal energy $u$, mass flux $w$, and the parting sheet temperature $T_{m}$ are the four dependent variables. Of course, the model PDEs must be applied to each fluid stream and parting sheet in the adiabatic group of the core, and the indexing convention, illustrated in Fig. 2, is used to distinguish them.

\section{Computer Implementation}

To obtain the numerical solution for our core heat exchanger model, we apply the Method of Lines to the model PDEs. That is, we divide the entire core in the axial direction into $N$ intervals of equal length, evaluate the spatial derivatives at the center of each interval by finite difference approximations and integrate the temporal derivatives at the center of 
each interval by an ordinary differential equation. integrator. Specifically we use the twopoint biased and three-point centered approximations implemented in the Fortran code DSS/2 (Ref. 3 ) to evaluate the first-order and second-order spatial derivatives, respectively, and the sparse matrix integrator implemented in the Fortran code LSODES (Ref. 4) to integrate the temporal derivatives. It is essential in the Method of Lines that the-temporal derivatives can be determined at any instant (including the initial moment) to allow their integration, i.e., the right-hand sides of the model PDEs must be computed. Thus we need to not only evaluate the spatial derivatives, but also compute all quantities, other than the four dependent variables, that appear in the right-hand sides of the equations. These quantities are $h, P, \mu, Q, Q_{m}$, $D, S, S_{m}, \rho_{m}, c_{m}$, and $k_{m}$. In the following discussion, the calculation of these quantities is detailed along with the specification of the initial and boundary conditions required to solve the model PDEs. Note that a simplified fin structure, shown in Figure 3, is adopted in our model to facilitate the analysis of heat exchange with the fins. As indicated in Fig. $3, h_{f}$ is the fin height, $b_{f}$ the fin thickness, and $n_{f}$ the number of fins per unit width of the core.
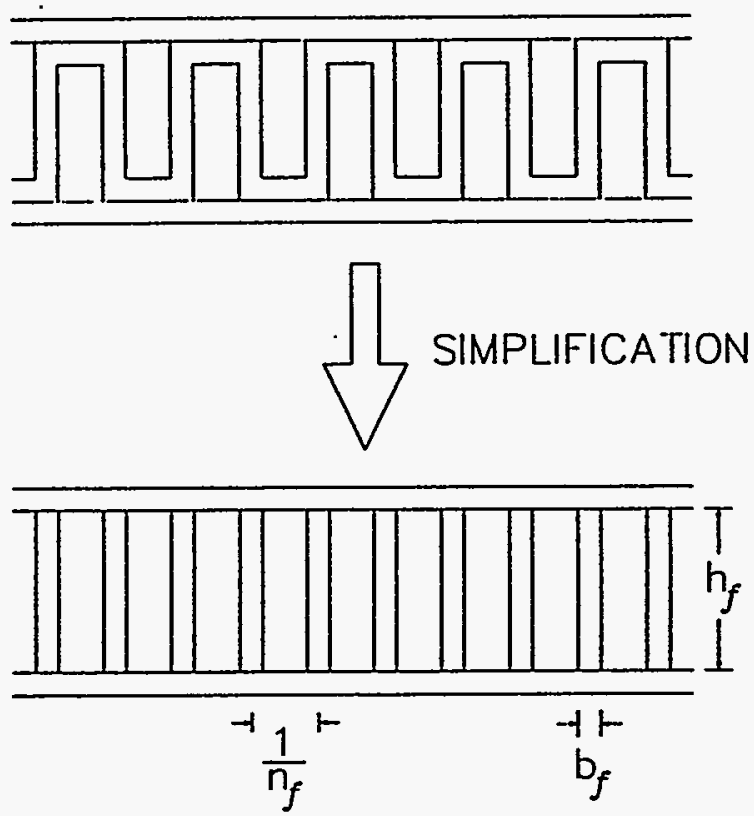

Figure 3: Simplification of the fin structure adopted in our model.

\section{Geometrical Quantities}

The flow cross-sectional area $S$, hydraulic diameter $D$, and parting sheet cross-sectional area $S_{m}$ are geometrical quantities. In terms of the fin parameters $b_{f}, h_{f}$ and $n_{f}$, the flow crosssectional area $S$ is given by

$$
S=w_{c} n_{f}\left(\frac{1}{n_{f}}-b_{f}\right) h_{f},
$$

where $w_{c}$ is the width of the core. The hydraulic diameter $D$ is defined to be four times the ratio of the flow cross-sectional area $S$ to the flow cross-sectional perimeter $C$ which, in terms of the fin parameters, is given by

$$
C=2\left(\frac{1}{n_{f}}-b_{f}+h_{f}\right) w_{c} n_{f} .
$$


In general different streams in a core heat exchanger have different fin parameters and thus different flow cross-sectional areas $S$ and hydraulic diameters $D$. The parting sheet crosssectional area $S_{m}$ is given by

$$
S_{m}=b_{m} w_{c}
$$

where $b_{m}$ is the parting sheet thickness. $S_{m}$ is. constant for each parting sheet.

\section{Other Thermodynamic Properties}

Given the He density $\rho$ and internal energy $u$ from Eqs. (1) and (4), we use a He thermodynamic model (Ref. 5) to obtain the He enthalpy $h$, pressure $P$ and temperature $T$. The He temperature $T$, as we will see below, is required to calculate the heat transfer rates $Q$ and $Q_{m}$. The He viscosity $\mu$ and the parting sheet density $\rho_{m}$ and specific heat $c_{m}$ are essentially constant in the range of the He thermodynamic properties under which the core heat exchangers are operated; suitable values have been used for each of them in the model. The parting sheet thermal conductivity $k_{m}$, however, varies significantly with temperature. This temperature dependence is taken into account in the model. Because of this, $k_{m}$ is inside the second derivative with respect to $z$ in Eq. (9).

\section{Heat Transfer Rates}

Figure 4 indicates the various heat fluxes that contribute to the cooldown and warmup of the $j$ th stream and parting sheet: $q_{1, j}$ is the heat flux from parting sheet $j$ to stream $j ; q_{2, j}$ is the heat flux from parting sheet $j+1$ to stream $j ; q_{3, j}$ is the heat flux from parting sheet $j$ to stream $j-1 ; q_{4, j}$ is the heat flux from the fins in stream $j$ to stream $j ; q_{5, j}$ is the heat flux from parting sheet $j$ to the fins in stream $j$; and $q_{6, j}$ is the heat flux from the fins in stream $j-1$ to parting sheet $j$. If $A_{1, j}, A_{2, j}, A_{3, j}, A_{4, j}, A_{5, j}$, and $A_{6, j}$ denote the corresponding heat transfer areas per unit length for these heat fluxes, the heat transfer rate per unit length to stream $j, Q_{j}$, and the heat transfer rate per unit length to parting sheet $j, Q_{m, j}$, are given by the following equations:

$$
Q_{j}=q_{1, j} A_{1, j}+q_{2, j} A_{2, j}+q_{4, j} A_{4, j}
$$

and

$$
Q_{m, j}=-q_{1, j} A_{1, j}-q_{3, j} A_{3, j}-q_{5, j} A_{5, j}+q_{6, j} A_{6, j} .
$$

Note that the above heat fluxes are directional, i.e., when their values are positive, the directions of heat flow are indicated by Fig. 4 and when their values are negative, the directions are reversed. With Fig. 4 in mind, the following equations can be written for the various heat fluxes:

$$
\begin{array}{ll}
q_{1, j}=h_{j}\left(T_{m, j}-T_{j}\right) & j=1,2, \ldots, M, \\
q_{2, j}=h_{j}\left(T_{m, j+1}-T_{j}\right) & j=1,2, \ldots, M-1, \\
q_{3, j}=h_{j-1}\left(T_{m, j}-T_{j-1}\right) & j=2,3, \ldots, M, \\
q_{4, j}=h_{j} \Delta T_{j} & j=1,2, \ldots, M, \\
q_{5, j}=-k_{m, j}\left(\frac{d T_{f, j}}{d x}\right)_{x=0} & j=1,2, \ldots, M,
\end{array}
$$




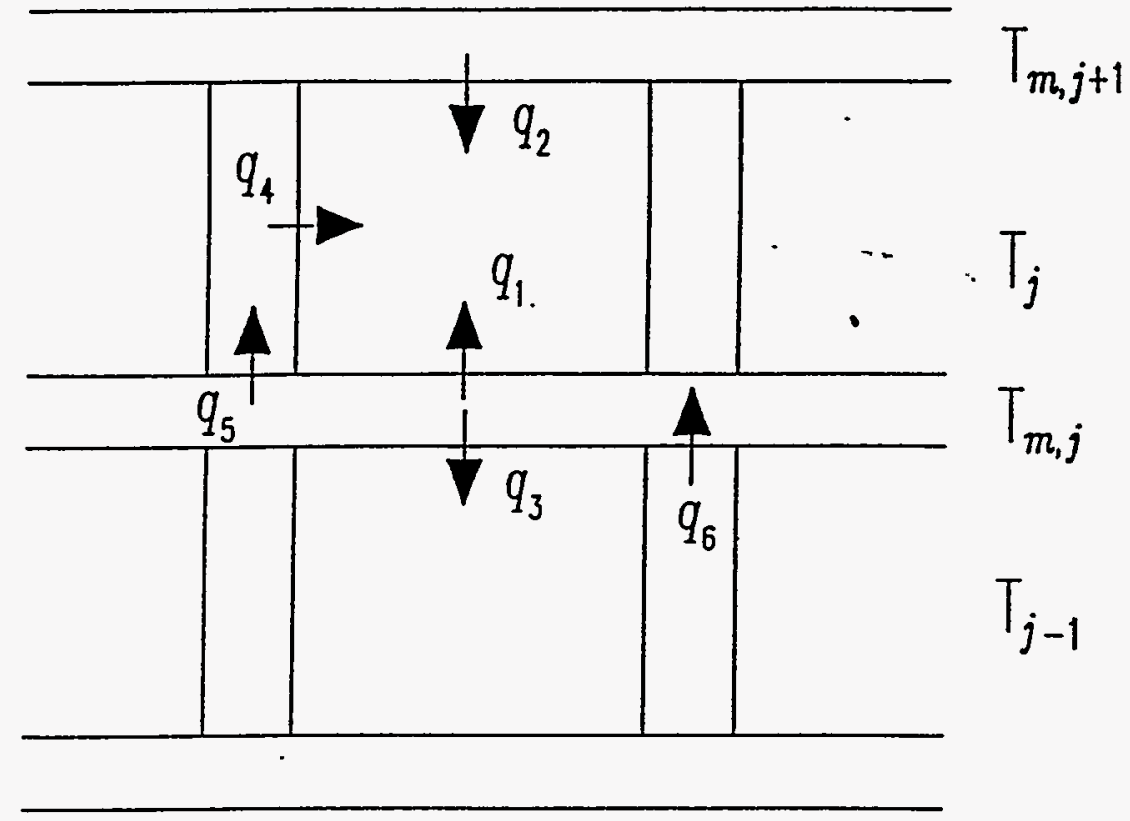

Figure 4: Various heat fluxes that contribute to the cooldown and warmup of the $j$ th stream and parting sheet.

and

$$
q_{6, j}=-k_{m, j}\left(\frac{d T_{f, j-1}}{d x}\right)_{x=h_{f, j-1}} \quad j=2,3, \ldots, M
$$

where $M$ is the number of different streams in the adiabatic group, $h_{j}$ the heat transfer coefficient of stream $j, \Delta T_{j}$ the average temperature difference between stream $j$ and the fins within stream $j$, and $T_{f, j}(x)$ the temperature of the fins between parting sheets $j$ and $j+1$ at position $x$ along the fin height. In the next section we derive $T_{f, j}(x)$ and define $\Delta T_{j}$. Periodic arrangement of the adiabatic group in the core and mirror symmetry inside the adiabatic group allow us to determine $q_{3, j}$ and $q_{6, j}$ for $j=1$ and $q_{2, j}$ for $j=M$ respectively,

$$
q_{2, j=M}=q_{1, j=M}
$$

and

$$
q_{3, j=1}=q_{1, j=1}, \quad q_{6, j=1}=-q_{5, j=1} .
$$

The associated heat transfer areas (again, referring to Fig. 4) are given by the following equations:

$$
\begin{array}{ll}
A_{1, j}=w_{c} n_{f, j}\left(\frac{1}{n_{f, j}}-b_{f, j}\right) & j=1,2, \ldots, M, \\
A_{2, j}=A_{1, j} & j=1,2, \ldots, M, \\
A_{3, j}=w_{c} n_{f, j-1}\left(\frac{1}{n_{f, j-1}}-b_{f, j-1}\right) & j=2,3, \ldots, M, \\
A_{4, j}=2 w_{c} n_{f, j} h_{f, j} & j=1,2, \ldots, M, \\
A_{5, j}=w_{c} n_{f, j} b_{f, j} & j=1,2, \ldots, M,
\end{array}
$$


and

$$
A_{6, j}=w_{c} n_{f, j-1} b_{f, j-1} \quad j=2,3, \ldots, M
$$

Again, the periodic arrangement of the adiabatic group gives $A_{3, j}$ and $A_{6, j}$ for $j=1$,

$$
A_{3, j=1}=A_{1, j=1}, \quad A_{6, j=1}=A_{5, j=1} \cdot \cdot
$$

Since we are given in the heat exchanger specifications the total heat transfer rates per degree for each stream $U_{j}$ (not the heat transfer coefficients $h_{j}$ ), the following equation is used to deduce $h_{j}$,

$$
h_{j}=\frac{U_{j}}{C_{j} L n_{j}}
$$

where $C_{j}$ is given by Eq. (11) for stream $j, L$ the length of of the core, and $n_{j}$ the total number of stream $j$ in the core.

\section{Fin Temperature Distribution}

Assuming that the fins between two parting sheets are in steady state at any instant, application of an energy balance to the fins gives

$$
\frac{d^{2} T_{f}}{d x^{2}}-\frac{2 h}{b_{f} k_{m}}\left(T_{f}-T\right)=0 .
$$

In deriving Eq. (31) we have assumed $k_{m}$ does not vary with $x$. The general solution of Eq. (31) is

$$
T_{f}=T+C_{1} \sinh (\alpha x)+C_{2} \cosh (\alpha x),
$$

where $\alpha=\sqrt{2 h / b_{f} k_{m}}$. Equation (32) should be applied to the fins in each stream. Using the boundary conditions $T_{f}(x=0)=T_{m, j}$ and $T_{f}\left(x=h_{f, j}\right)=T_{m, j+1}$ for the fins in stream $j$, we determine $C_{1, j}$ and $C_{2, j}$ as follows,

$$
C_{1, j}=\frac{\left(T_{m, j+1}-T_{j}\right)-\left(T_{m, j}-T_{j}\right) \cosh \left(\alpha h_{f, j}\right)}{\sinh \left(\alpha h_{f, j}\right)} \quad j=1,2, \ldots, M-1
$$

and

$$
C_{2, j}=T_{m, j}-T_{j} \quad j=1,2, \ldots, M .
$$

The mirror symmetry inside the adiabatic group gives $C_{1, j}$ for $j=M$,

$$
C_{1, j=M}=\left.\frac{\left(T_{m, j}-T_{j}\right)-\left(T_{m, j}-T_{j}\right) \cosh \left(\alpha h_{f, j}\right)}{\sinh \left(\alpha h_{f, j}\right)}\right|_{j=M}
$$

The fin-to-stream heat flux $q_{4}$ is determined from the following expression,

$$
q_{4}=\frac{2 w_{c} n_{f}\left(\int_{0}^{h_{f}} h\left(T_{f}-T\right) d x\right)}{2 w_{c} n_{f} h_{f}},
$$

i.e., the ratio of the total heat transfer rate per unit length of the core to the total heat transfer area per unit length. Since $q_{4}=h \Delta T$ by definition, we have

$$
\Delta T=\frac{1}{h_{f}} \int_{0}^{h_{f}}\left(T_{f}-T\right) d x,
$$


which is the average of the temperature difference $T_{f}-T$ over the fin height $h_{f}$. It is straightforward to obtain the following expressions from Eq. (32):

$$
\begin{gathered}
\Delta T=\frac{1}{\alpha h_{f}}\left\{C_{1} \cosh \left(\alpha h_{f}\right)+C_{2} \sinh \left(\alpha h_{f}\right)-C_{1}\right\}, \\
\left(\frac{d T_{f}}{d x}\right)_{x=0}=C_{1} \alpha
\end{gathered}
$$

and

$$
\left(\frac{d T_{f}}{d x}\right)_{x=h_{f}}=C_{1} \alpha \cosh \left(\alpha h_{f}\right)+C_{2} \alpha \sinh \left(\alpha h_{f}\right) .
$$

Equations (38), (39) and (40) are used in Eqs. (18), (19) and (20) to compute $q_{4}, q_{5}$ and $q_{6}$, respectively.

\section{Initial and Boundary Conditions}

For boundary conditions, we have chosen to specify the inlet temperature and pressure and the outlet pressure for the He streams. Also we use the insulated (Neumann) boundary condition at both ends of the parting sheets, i.e.,

$$
\left(\frac{\partial T_{m}}{\partial z}\right)_{z=0}=\left(\frac{\partial T_{m}}{\partial z}\right)_{z=L}=0
$$

to specify that the heat leak from the ends of the parting sheets is negligible. The inlet mass flux is treated as another time-dependent variable whose temporal behavior is governed by the momentum balance equation, Eq. (7). The spatial derivatives $\partial w / \partial z, \partial h / \partial z$, and $\partial\left(w^{2} / \rho\right) / \partial z$ are approximated by two-point upwind finite differences, and thus the inlet conditions are built into the approximations. The spatial derivative $\partial P / \partial z$ is approximated by two-point downwind finite differences, and thus the outlet pressure is built into the approximations. In applying the momentum balance to compute the inlet mass flux, the spatial derivative $\partial\left(w^{2} / \rho\right) / \partial z$ at the inlet is assumed to be the same as that at the first grid point (the center of the first spatial interval) downstream. If the adiabatic group has $M$ different streams and the length of the core is divided into $N$ intervals, applying the Method of Lines to the four model PDEs then requires integration of $4 M N+M$ temporal ODEs.

To provide a consistent initial condition for the dynamic model, a steady state calculation is included. This is done by setting the temporal derivatives in the model PDEs to zero, approximating the spatial derivatives by the same finite difference methods as used in the dynamic model, and solving the resulting algebraic equations with the IMSL subroutine DNEQNF (Ref. 6). If uniform mass flux is assumed, i.e., the temporal derivative in the continuity equation is automatically set to zero, only three temporal derivatives, i.e., $\partial u / \partial t, \partial w / \partial t$ and $\partial T_{m} / \partial t$, are required to be zero. For $M$ different streams in the adiabatic group and $N$ axial intervals in the core, we then need to solve $3 M N+M$ nonlinear algebraic equations for $3 M N+M$ unknowns which are $\rho, u$ and $T_{m}$ at the center of each spatial interval and the steady state mass flux or the outlet pressure for each stream.

A scaling factor $s_{f}$ is used to adjust the friction factor $f$ to achieve realistic pressure drops in the core heat exchangers, since the Blasius formula for friction factor, Eq. (8), which is valid for smooth pipes, gives unrealistically small pressure drops. Typical values of the scaling factor are about 10 from our simulation, which are well below 40 , the upper bound for realistic designs (Ref. 7). Because the steady state mass flux, friction scaling factor and outlet pressure 
are interrelated, our model allows that any two of these three variables can be specified in the steady state calculation and the third will be determined by the IMSL nonlinear equation solver. The scaling factor used or determined in the steady state calculation is assumed to remain the same in the dynamic simulation.

\section{Simulation Results}

To check the code for our static and dynamic core heat exchanger models, we have simulated heat exchanger $\mathrm{R} 2$ in a SSC 4K prototype refrigeration plant (Ref. 8). The passage arrangement of heat exchanger $R 2$ is the adiabatic group $B A B$ repeated 18 times where $A$ is the high pressure He stream flowing down and $B$ the low pressure He return stream flowing up. Listed below are the parameters for $\mathrm{R} 2$ required as input to the model:

\begin{tabular}{cccl}
\hline \hline Parameter & Stream A & Stream B & Unit \\
\hline$b_{f}$ & 0.010 & 0.008 & in \\
$h_{f}$ & 0.281 & 0.380 & in \\
$n_{f}$ & 20.2 & 14.7 & in $^{-1}$ \\
$U$ & 96321 & 96319 & $\mathrm{BTU}^{\mathrm{h}} \mathrm{hr}-{ }^{\circ} \mathrm{F}$ \\
$L=10 \mathrm{ft}$ & & & \\
$w_{c}=16.75 \mathrm{in}$ & & & \\
$b_{m}=0.032 \mathrm{in}$ & & & \\
\hline \hline
\end{tabular}

The following data on temperature, pressure and mass flux are used in the initial steady state calculation:

\begin{tabular}{crrl}
\hline \hline Parameter & \multicolumn{1}{c}{ Stream A } & \multicolumn{1}{c}{ Stream B } & Unit \\
\hline$P_{\text {in }}$ & 264.00 & 11.17 & psia \\
$T_{\text {in }}$ & -315.69 & -417.63 & ${ }^{\circ} \mathrm{F}$ \\
$P_{\text {out }}$ & 263.96 & 10.94 & $\mathrm{psia}$ \\
$w$ & -4361 & 1437 & $\mathrm{lb} / \mathrm{hr}-\mathrm{ft}^{2}$ \\
\hline \hline
\end{tabular}

where the subscripts in and out refer to the inlet and outlet streams, respectively. The friction scaling factor, as determined from the IMSL nonlinear equation solver, is 8.685 for stream $\mathrm{A}$ and 9.741 for stream $B$.

We simulated the unsteady state resulting from increasing the inlet temperature of stream A by $10^{\circ} \mathrm{F}$. The simulation results for $20 \mathrm{sec}$ are shown in Figures 5,6 and 7, i.e., the axial profiles of mass fiux, temperature and pressure, respectively. We see from Figure 5 that the mass flux of each stream varies from one uniform value to another as time passes, indicating that starting with one steady state we approach another; Figure 6 indicates that the temperatures of both streams increase at the inlet of stream A but do not change at the inlet of stream B, as expected. It is interesting to note in Figure 7 that the pressure of stream $A$ decreases and then rises in the direction of the flow. This results from our inclusion of the gravitational force in the momentum balance equation. 
STREAM A

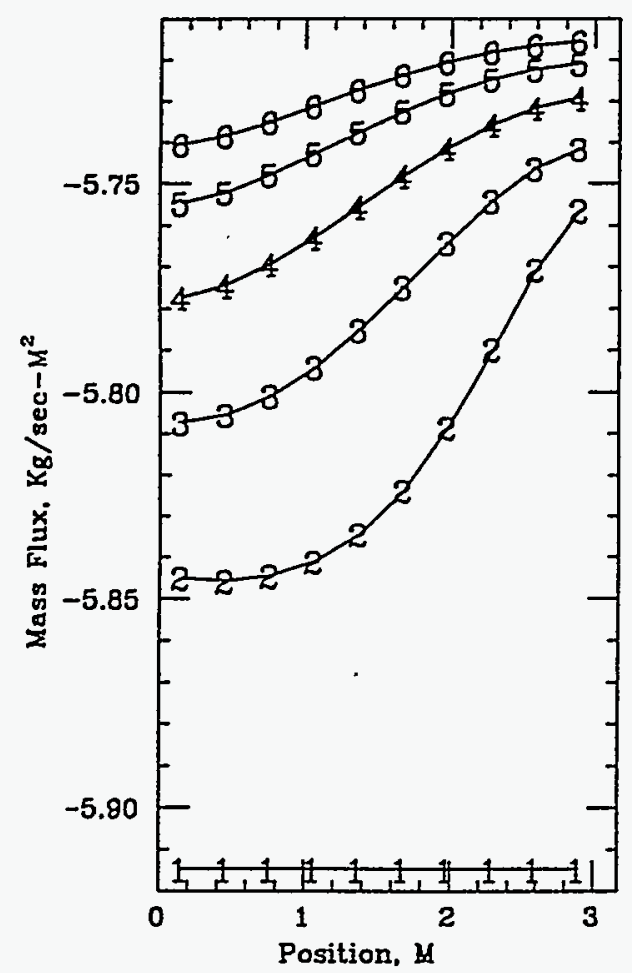

STREAM B

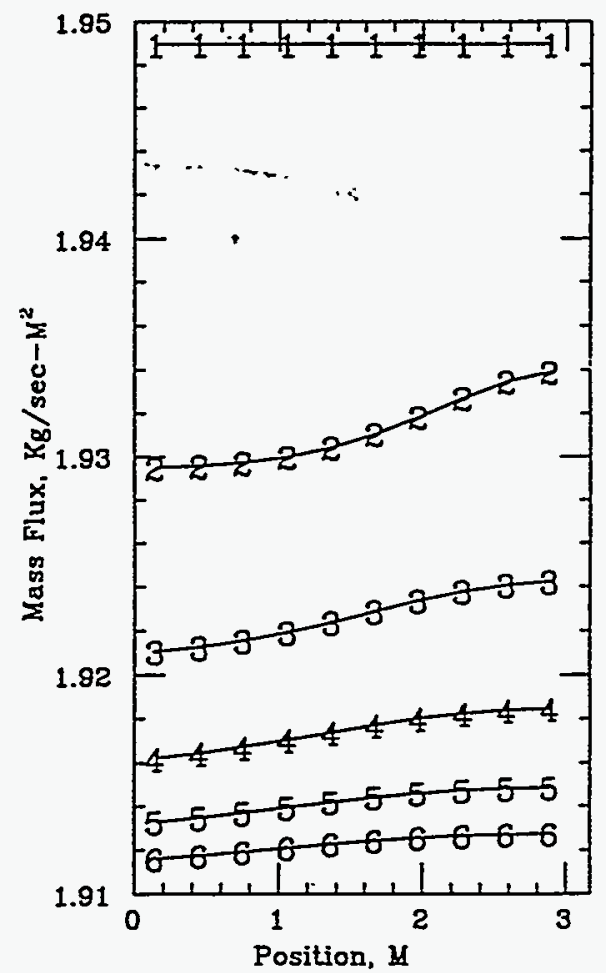

Figure 5: Axial profiles of the stream mass flux. Each profile is 4 seconds apart. The curve index increases with time.
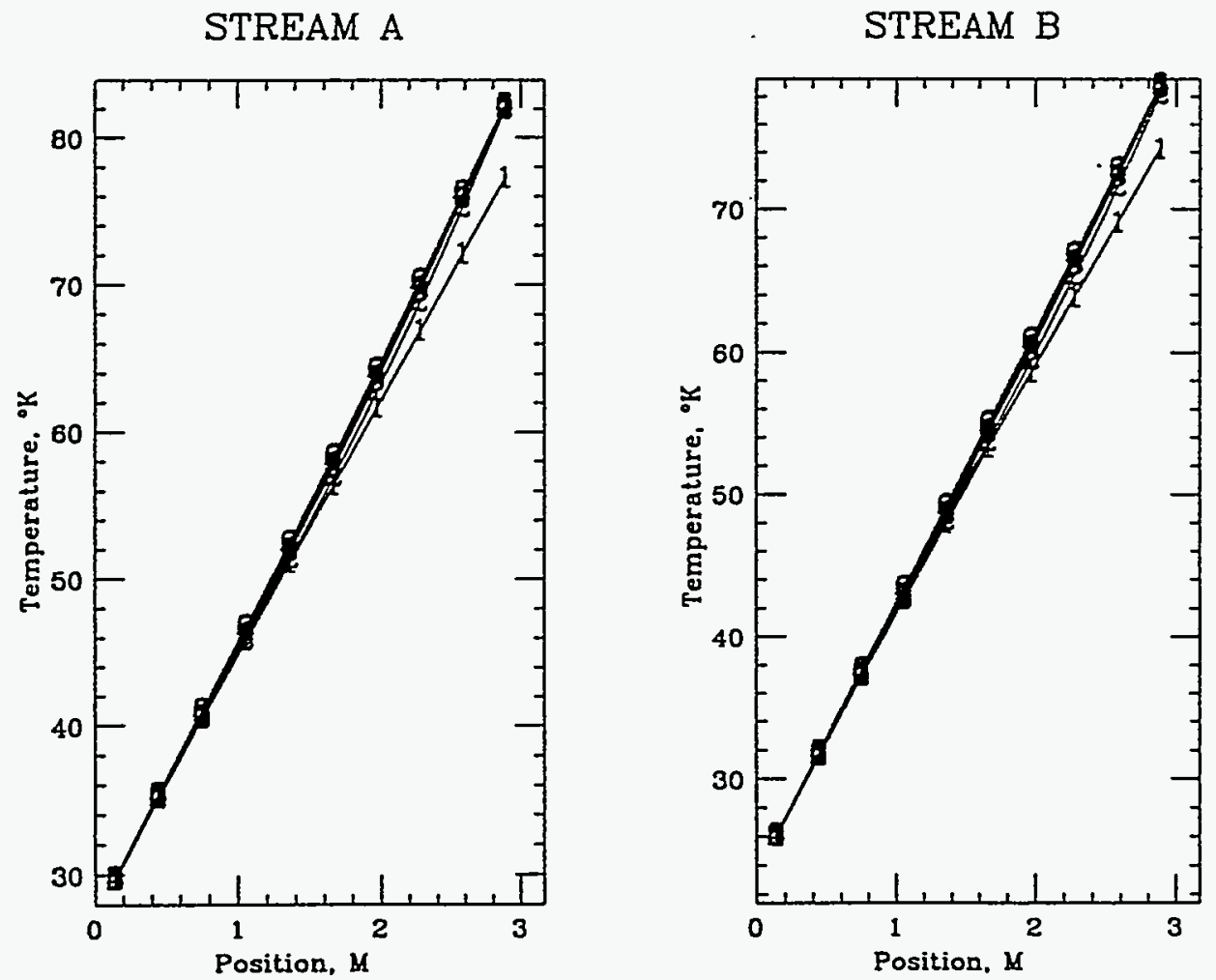

Figure 6: Axial profiles of the stream temperature. Each profile is 4 seconds apart. The curve index increases with time. 
STREAM A

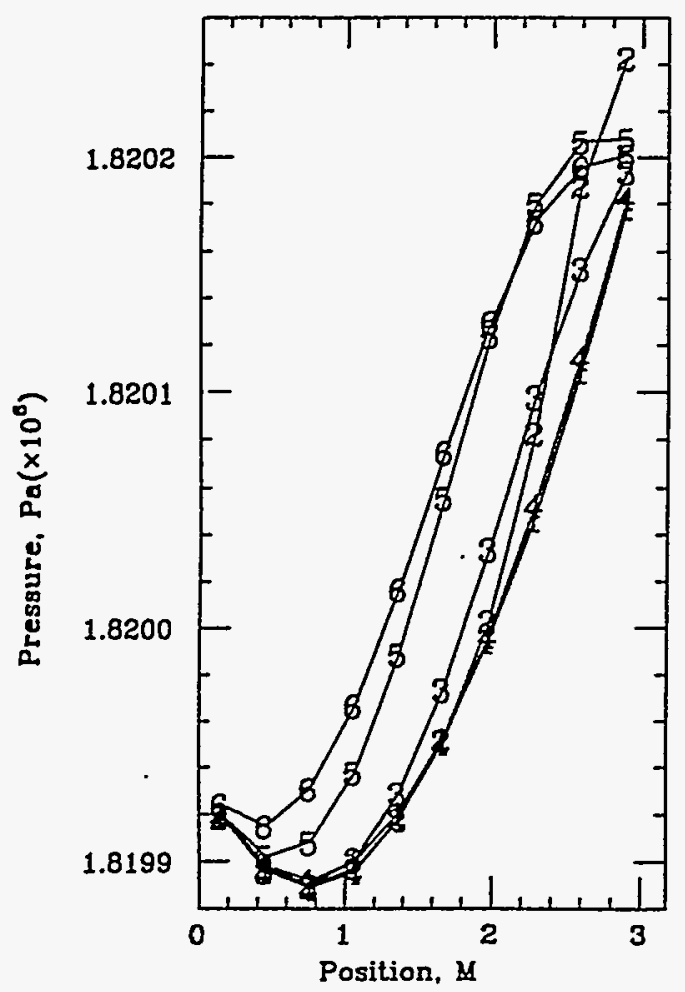

STREAM B

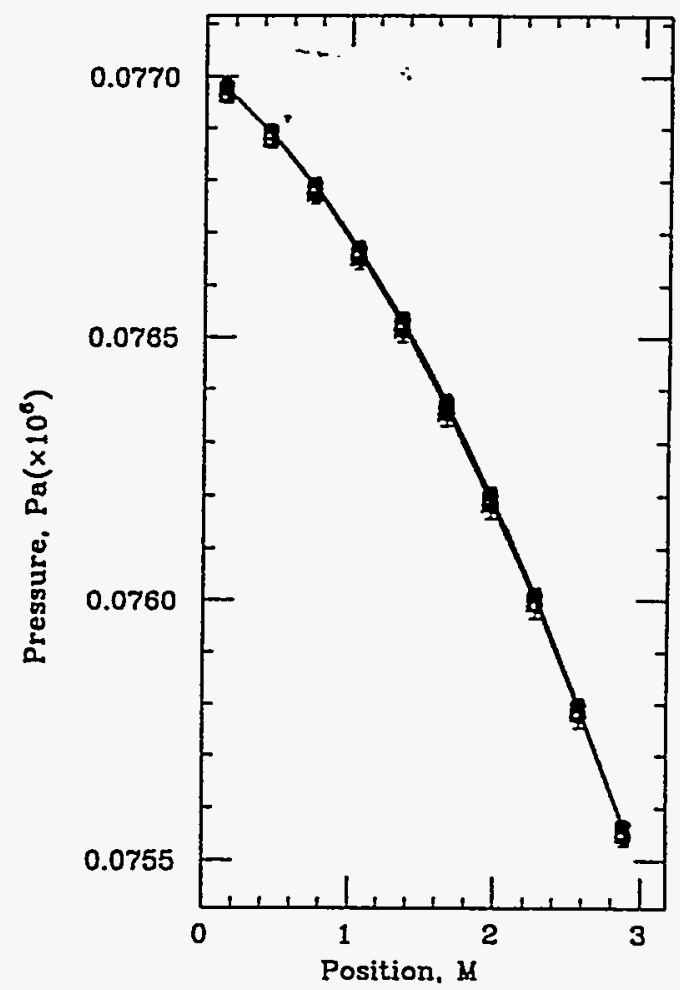

Figure 7: Axial profiles of the stream pressure. Each profile is 4 seconds apart. The curve index increases with time.

We found that it was important to restrict the order of the algorithm in the ODE integrator LSODES. Specifically, we have found that by restricting the maximum order of the algorithm of LSODES to two (instead of the usual upper limit of five) the computer time is greatly reduced (by two orders of magnitude). This suggests that the greatly increased efficiency of the ODE integration by LSODES is due to the existence of nearly imaginary eigenvalues of the ODE system. For the BDF (Gear) methods on which LSODES is based, the order three and above algorithms have poor stability near the imaginary axis (Ref. 9), and will therefore take small steps if the ODE system has nearly imaginary eigenvalues; computing the temporal eigenvalues of our model ODE system indicated this is the case.

\section{Reduced Model}

Using the simulation results from our rigorous core heat exchanger model, we developed an approximate low-order model representation (Ref. 10) which could be used when the execution times of the rigorous dynamic model are excessive, e.g., when several heat exchangers are included in a complete refrigeration plant simulation. The low-order model relates the following inputs and outputs: 


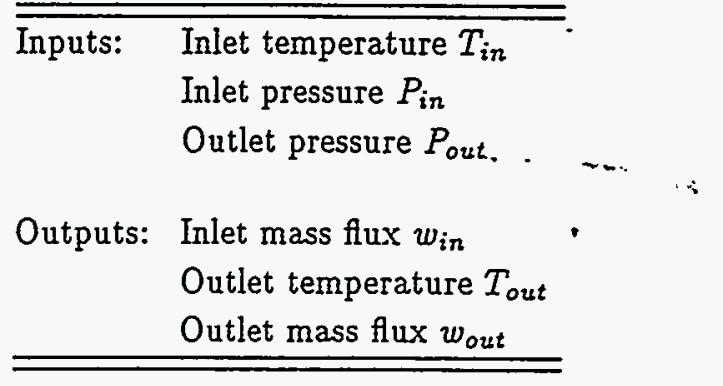

The procedure generally involves estimating the time constants of the inlet mass flux, outlet temperature and outlet mass flux responses for each stream from rigorous static and dynamic simulations, then approximating the transient response of these three state variables with single exponentials (first-order model) for a series of step inputs. The details are illustrated by the following example calculation for heat exchanger $R 2$.

Let $X$ denote one of the three output variables $w_{i n}, T_{\text {out }}$ and $w_{\text {out }}$. From the steady state calculation of our rigorous model, we obtain $X_{i}$ and $X_{f}$, the initial and final values of $X$, for a given (arbitrary) step input. Assuming the transient $X(t)$ is governed by a single exponential with a time constant $t_{c}$, we calculate the value of $X$ at $t=t_{c}$ according to

$$
X_{c}=X_{i}+0.362\left(X_{f}-X_{i}\right) \text {. }
$$

From the rigorous dynamic simulation we obtain two consecutive times $t_{s}$ and $t_{s+1}(\Delta t=$ $t_{s+1}-t_{s}$ should be reasonably small) such that $X_{s}<X_{c}<X_{s+1}$ or $X_{s+1}<X_{c}<X_{s}$, where $X=X_{s}$ at $t=t_{s}$ and $X=X_{s+1}$ at $t=t_{s+1}$. The time constant $t_{c}$ is then obtained by linear interpolation, i.e.,

$$
t_{c}=t_{s}+\frac{\left(X_{c}-X_{s}\right)}{\left(X_{s+1}-X_{s}\right)}\left(t_{s+1}-t_{s}\right)
$$

Listed below are the time constants (in seconds) determined this way for heat exchanger $\mathrm{R} 2$ for a step increase in the inlet temperature of stream $\mathrm{A}$ of $10^{\circ} \mathrm{F}$ :

\begin{tabular}{lcrr}
\hline \hline & $w_{\text {in }}$ & \multicolumn{1}{c}{$T_{\text {out }}$} & $w_{\text {out }}$ \\
\hline Stream A & 0.9 & 22.4 & 12.0 \\
Stream B & 7.2 & 1.7 & 9.1 \\
\hline \hline
\end{tabular}

To compute the response of the first-order model to a general time-varying input, we first approximate the input by a series of step functions, then we obtain the final steady state for each step change from the steady state solution of our rigorous model. With the time constants determined above, the transient response for each time step, denoted with a superscript $(s)$, is computed according to:

$$
X^{(s)}=\left(X_{i}^{(s)}-X_{f}^{(s)}\right) \exp \left(-\frac{\left(t-t^{(s)}\right)}{t_{c}}\right)+X_{f}^{(s)}
$$

where $t^{(s)}$ is the time at the beginning of step $(s)$. The above formula is just the solution of the following linear ODE:

$$
\frac{d X^{(s)}}{d t}=-\frac{1}{t_{c}}\left(X^{(s)}-X_{f}^{(s)}\right), \quad X^{(s)}\left(t^{(s)}\right)=X_{i}^{(s)} .
$$


Thus each of the three outputs is described in the reduced model by a linear ODE. Figures 8 , $\dot{g}$ and 10 show the transient responses of $w_{\text {in }}, T_{\text {out }}$ and $w_{\text {out }}$ from the first-order model for a series of step changes in the inlet temperature of stream $\mathrm{A}:+5^{\circ} \mathrm{F}$ at $t=0 \mathrm{sec},+5^{\circ} \mathrm{F}$ at $t=5 \mathrm{sec}$, and $-10^{\circ} \mathrm{F}$ at $t=15 \mathrm{sec}$. For comparison, the transient responses from the rigorous model subject to the same input are also shown. In general this reduced model works rather well considering it reduces a large number of nonlinear ODEs $(4 M N+M)$ to a small number of linear ODEs $(3 M)$. It has the major advantage that it is always correct at steady state (by "correct," we mean that it agrees with the rigorous model at steady state).

In principle, this model reduction technique can be applied to any of the core heat exchangers, i.e., it appears to be a general methodology, although further testing is needed to establish its general utility. Also, the use of the method presupposes that the steady state calculations can be done fast enough to compute the series of $X_{f}$ required along the transient. This in turn assumes the iterative solution of the nonlinear algebraic equations by subroutine DNEQNF will converge rapidly. This convergence will be enhanced by the fact that for relatively small steps, $X_{f}$ will not be too far from $X_{i}$, and therefore, $X_{i}$ serves as a reasonably good initial guess for DNEQNF. However, we cannot guarantee in advance that the model reduction technique, or for that matter, the rigorous dynamic simulation code, will produce a solution. Much depends on the success of the steady state calculation with DNEQNF, and the solution of large systems of nonlinear algebraic equations is a notoriously difficult computational problem. The choice of the initial estimate of the solution is critical in this process.

\section{STREAM A}

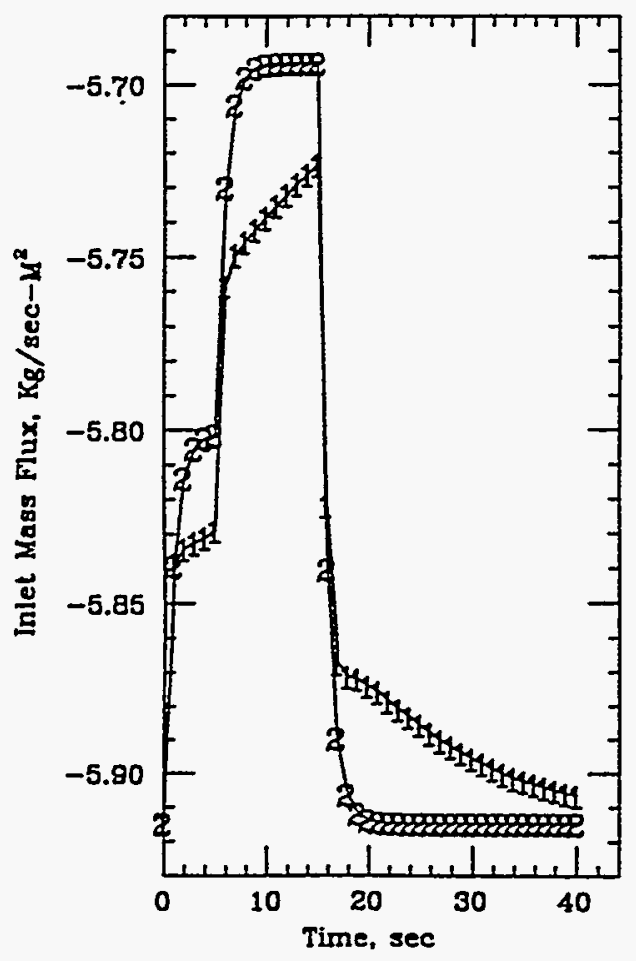

STREAM B

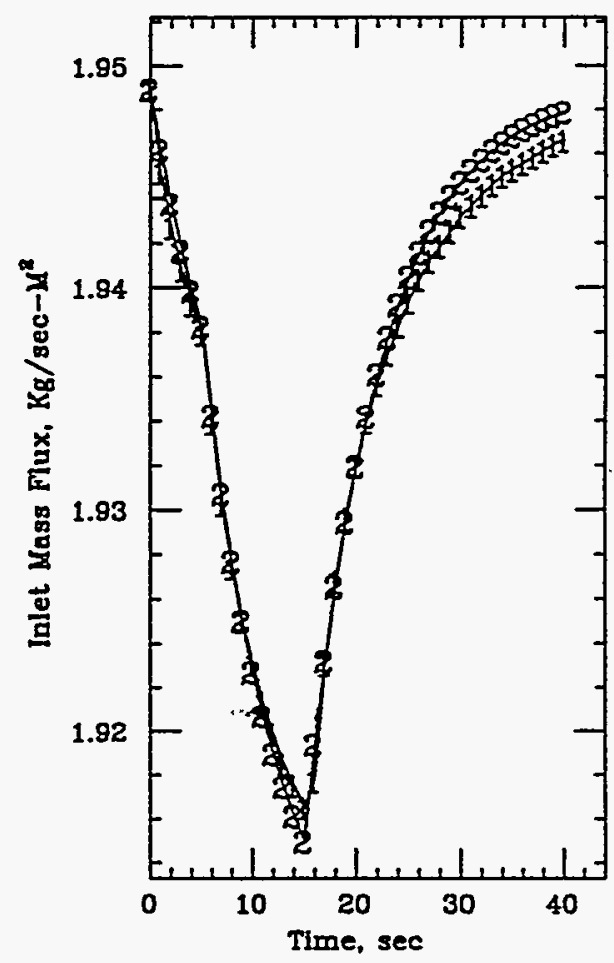

Figure 8: Transient responses of the inlet mass flux for a series of step changes in the inlet temperature of stream $\mathrm{A}:+5^{\circ} \mathrm{F}$ at $t=0 \mathrm{sec},+5^{\circ} \mathrm{F}$ at $t=5 \mathrm{sec}$, and $-10^{\circ} \mathrm{F}$ at $t=15 \mathrm{sec}$. Curve 1 is from the rigorous model and curve 2 from the reduced model. 
STREAM A

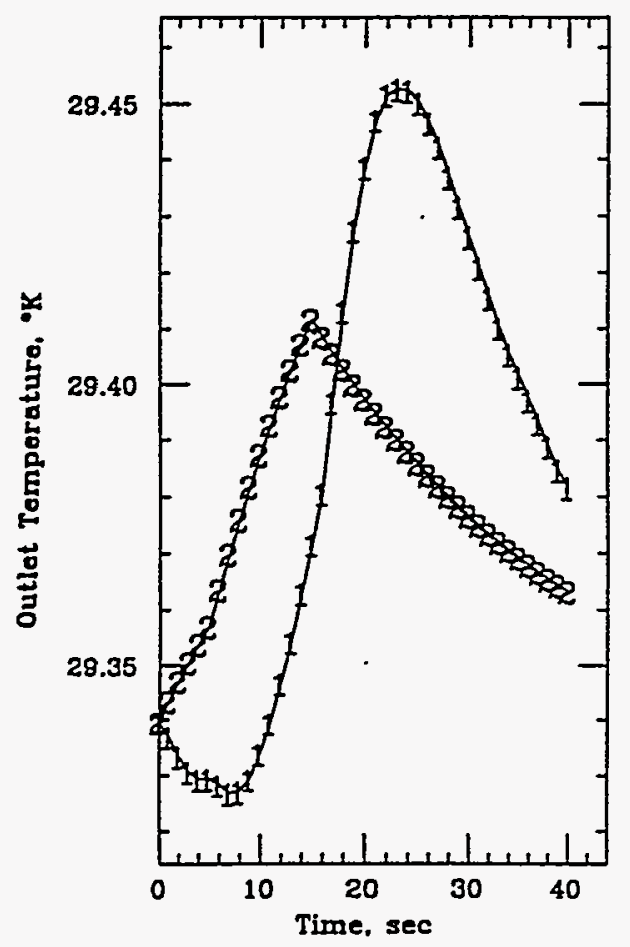

STREAM B

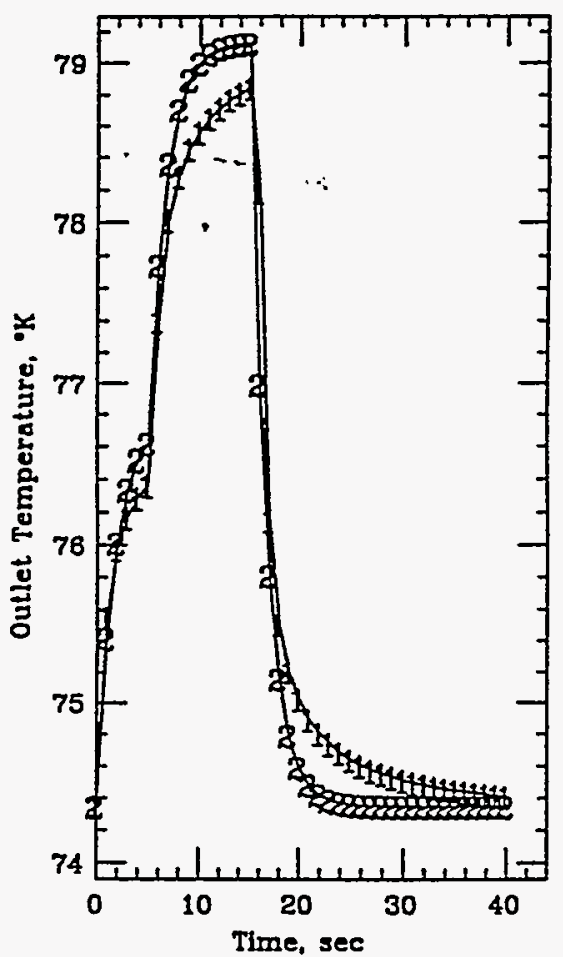

Figure 9: Transient responses of the outlet temperature for a series of step changes in the inlet temperature of stream A: $+5^{\circ} \mathrm{F}$ at $t=0 \mathrm{sec},+5^{\circ} \mathrm{F}$ at $t=5 \mathrm{sec}$, and $-10^{\circ} \mathrm{F}$ at $t=15 \mathrm{sec}$. Curve 1 is from the rigorous model and curve 2 from the reduced model.

STREAM A

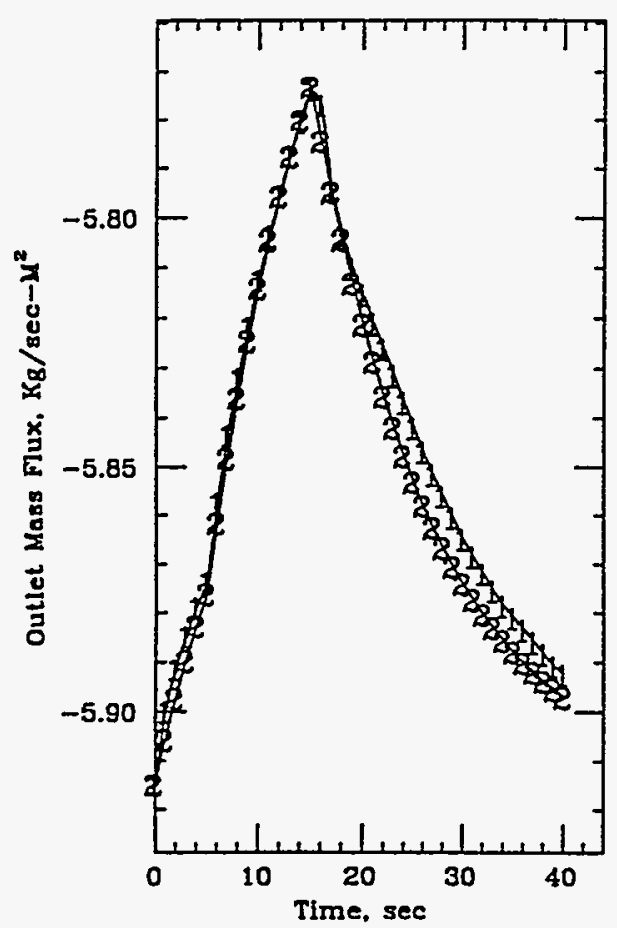

STREAM B

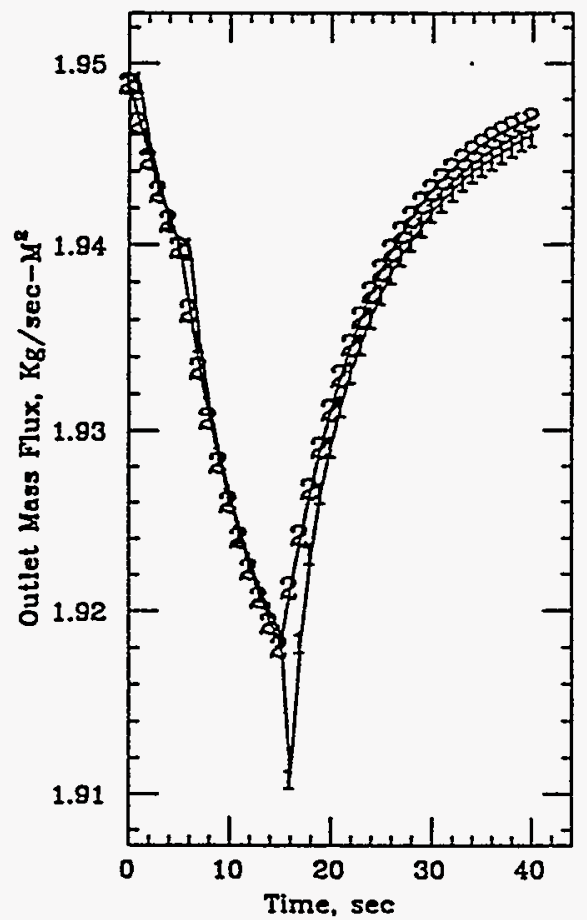

Figure 10: Transient responses of the outlet mass flux for a series of step changes in the inlet temperature of stream $\mathrm{A}:+5^{\circ} \mathrm{F}$ at $t=0 \mathrm{sec},+5^{\circ} \mathrm{F}$ at $t=5 \mathrm{sec}$, and $-10^{\circ} \mathrm{F}$ at $t=15 \mathrm{sec}$. Curve 1 is from the rigorous model and curve 2 from the reduced model. 
Conclusions

A detailed model for He core heat exchangers has been described in this paper. The associated Fortran 77 implementation has a user-selected flag for the following options:

(1) A dynamic model consisting of a system of partial differential-equations expressing the conservation of mass, momentun and energy for the He and the conservation of energy for the parting sheets. Time and position along the exchanger are the independent variables of this model. The time integration is performed by a sparse matrix implementation of the backward differentiation formulas; the spatial derivatives in the PDEs are approximated by upwind and downwind finite differences.

(2) A steady state model obtained by setting the temporal (time) derivatives of the dynamic model, (1), to zero. The resulting system of nonlinear algebraic equations is solved by Newton iteration. In particular, this steady state model provides a consistent initial condition for the dynamic model, (1).

(3) An approximate, low-order model, for the dynamic model, (1), which can be used in simulation and control studies.

Special features of (1) and (2) include: (a) a choice of rigorous and ideal gas He thermodynamic properties (the latter can often be used with good accuracy to save computer time), (b) detailed analysis of the thermal circuits which leads to six heat flux terms in the He energy balance, and (c) a modified Blasius pressure drop correlation with scaling computed by the code to account for the complex flow patterns within the exchanger.

The output from the dynamic model, in particular, gives: (a) the He state variables, e.g., temperature, pressure, density, enthalpy and internal energy, (b) the He mass flux, and (c) the parting sheet temperature as a function of time and position along the exchanger. Additionally, the six heat fluxes are available as a function of time and position which provide insight into the point-to-point operating characteristics within the exchanger. These variables can be calculated for any number of fluid streams and parting sheets within an adiabatic group; therefore, we anticipate the model and code can be used to study the steady state and dynamic performance of any of the heat exchangers in a SSC refrigeration plant (Ref. 8).

\section{References}

1. M. S. McAshan, "Refrigeration Plants for the SSC," SSC Central Design Group Report SSC-129, May 1987.

2. R. B. Bird et. al., Transport Phenomena, John Wiley \& Sons, New York (1960).

3. J. C. Pirkle, Jr. and W. E. Schiesser, "DSS/2: A Transportable Fortran 77 Program for Ordinary and One, Two, and Three-Dimensional Partial Differential Equations," Proceedings of the 1987 Summer Computer Simulation Conference, Montreal, July 1987.

4. A. C. Hindmarsh, "ODEPACK, A Systematized Collection of ODE Solvers," in Scientific Computing, R. S. Stepleman et. al. (eds.), North-Holland, Amsterdam (1983).

5. "Helium Thermodynamic System," Report by Air Products and Chemicals, Inc. to the SSC Central Design Group, March 1988.

6. IMSL MATH/LIBRARY - Fortran Subroutines for Mathematical Applications, Version 1.1, User's Manual, Vol. 2, January 1989.

7. D. M. Herron, Air Products and Chemicals, Inc., private communication. 
8. "SSC Site-Specific Conceptual Design Report," Vol. 1, Superconducting Super Collider Laboratory, Dallas, Texas, p. 447, December 1989.

9. C. W. Gear, Numerical Initial Value Problems in Ordinary Differential Equations, PrenticeHall, Englewood Cliffs (1971).

10. D. G. Hartzog, Air Products and Chemicals, Inc., private communication. 